\title{
Adaptive Stabilization Control for a Class of Complex Nonlinear Systems Based on T-S Fuzzy Bilinear Model
}

\author{
Jinsheng Xing and Naizheng Shi \\ School of Mathematics \& Computer Science, Shanxi Normal University, Linfen 041004, China \\ Correspondence should be addressed to Jinsheng Xing; xjs6448@126.com
}

Received 24 July 2014; Accepted 15 December 2014

Academic Editor: Lakshmanan Shanmugam

Copyright ( 92015 J. Xing and N. Shi. This is an open access article distributed under the Creative Commons Attribution License, which permits unrestricted use, distribution, and reproduction in any medium, provided the original work is properly cited.

\begin{abstract}
This paper proposes a stable adaptive fuzzy control scheme for a class of nonlinear systems with multiple inputs. The multiple inputs T-S fuzzy bilinear model is established to represent the unknown complex systems. A parallel distributed compensation (PDC) method is utilized to design the fuzzy controller without considering the error due to fuzzy modelling and the sufficient conditions of the closed-loop system stability with respect to decay rate $\alpha$ are derived by linear matrix inequalities (LMIs). Then the errors caused by fuzzy modelling are considered and the method of adaptive control is used to reduce the effect of the modelling errors, and dynamic performance of the closed-loop system is improved. By Lyapunov stability criterion, the resulting closed-loop system is proved to be asymptotically stable. The main contribution is to deal with the differences between the T-S fuzzy bilinear model and the real system; a global asymptotically stable adaptive control scheme is presented for real complex systems. Finally, illustrative examples are provided to demonstrate the effectiveness of the results proposed in this paper.
\end{abstract}

\section{Introduction}

Takagi-Sugeno (T-S) model-based fuzzy control is an effective and flexible tool for control of nonlinear systems. It has attracted wide attention [1-6]. The robust fuzzy control and adaptive fuzzy control approaches based on T-S fuzzy linear model with parameters uncertainties have been extensively studied in $[2,7-9]$ and the references therein. The adaptive fuzzy control based on T-S fuzzy model is divided into adaptive control based on static T-S fuzzy model (or fuzzy logic systems) and adaptive control based on dynamic T-S fuzzy model. Up to now, many results for the adaptive control based on fuzzy logic system have appeared. Compared with the adaptive control based on fuzzy logic system, the development of the adaptive control based on dynamic T-S fuzzy model is relatively slow; until recent years, the latter has become a focus issue in the control community. Novel research results have emerged in [10-19]. A novel direct T-S fuzzy neural online modelling and control method for a class of nonlinear systems with parametric uncertainties has been proposed, which utilized T-S fuzzy neural model to approximate the virtual linear system and designed the online identification algorithm and robust adaptive tracking controller in
$[10,11]$, respectively. Then the stability of the resulting closedloop systems was proved by Lyapunov stability criterion. In [13], the adaptive output tracking control problem of parameter strict-feedback system was discussed and the concept of virtual variable also was proposed based on LMI. In [14], the problem of simultaneously estimating the state and unknown inputs was considered in T-S fuzzy systems; sufficient conditions were given for the stability of the observer. The computation of the observer gains was based on solving a series of LMIs. In [17], a T-S fuzzy model is proposed to represent the nonlinear model of microelectromechanical systems gyroscope; a robust adaptive sliding mode control with online identification for the upper bounds of external disturbances and an adaptive estimator for the model uncertainty parameters are proposed in the Lyapunov framework. In [18], a new normal form of a global noncanonical form T-S fuzzy model was derived, and a new solution framework was developed for adaptive control of general discrete-time state-space T-S fuzzy systems with a relative degree. In [19], a novel design scheme of stable adaptive fuzzy control for a class of nonlinear systems was proposed. The parallel distributed compensation (PDC) method was utilized to design the fuzzy controller without the fuzzy modelling error. The sufficient conditions 
with respect to decay rate $\alpha$ were derived by linear matrix inequalities (LMIs) and by small-gain theorem. The adaptive compensation term was adopted to reduce the effect of the modelling. However, the consequence parts of the above T-S fuzzy models [10-19] were linear dynamic model or approximate linear dynamic model, so these methods have inevitable defects for some nonlinear systems.

It is known that bilinear models can describe many physical systems and dynamical processes in engineering fields $[20,21]$. There are two main advantages of the bilinear system. One is that it provides a better approximation to a nonlinear system than a linear one, and another is that many real physical processes may be appropriately modelled as bilinear systems when the linear models are inadequate. Considering the advantages of bilinear systems and T-S fuzzy control, the fuzzy control based on the T-S fuzzy model with bilinear rule consequence attracted the interest of researchers [22-26]. The T-S fuzzy bilinear model may be suitable for some classes of nonlinear plants. The robust stabilization for continuoustime fuzzy system with local bilinear model was studied in [22], and then the result was extended to the fuzzy system with time-delay only in the state [23]. The problem of robust stabilization for discrete-time fuzzy system with local bilinear model was investigated in [24]. Reference [25] focuses on the problem of nonfragile guaranteed cost control for a class of T$\mathrm{S}$ discrete-time fuzzy bilinear systems. Based on the parallel distributed compensation approach, the sufficient conditions were derived such that the closed-loop system was asymptotically stable and the cost function value was no more than a certain upper bound in the presence of the additive controller gain perturbations. In [26], an observer-based fuzzy control design was given for discrete-time T-S fuzzy bilinear systems. In $[27,28]$, authors proposed robust stability conditions for stochastic fuzzy impulsive recurrent neural networks with time-varying delays and uncertain stochastic fuzzy recurrent neural networks with mixed time-varying delays.

However, when there are differences between T-S fuzzy bilinear model and reality systems, these results will not be applied.

Considering the differences of the fuzzy model and the reality systems, in the paper, a stable adaptive fuzzy control for complex nonlinear systems is presented based on multiple inputs T-S fuzzy bilinear system with parameters uncertainties. In consideration of the modelling error, an adaptive fuzzy control is proposed to compensate for the issues. At first, the concept of the so-called PDC and LMI approach is employed to design the state feedback controller without considering the error caused by fuzzy modelling. The sufficient conditions with respect to decay rate $\alpha$ are derived in the sense of Lyapunov asymptotic stability. Then the error caused by fuzzy modelling is considered; an adaptive compensation term is designed to reduce the effect of the modelling error. The contributions of this paper are as follows: (i) the differences between T-S fuzzy bilinear model and the real system are considered in the modelling and analysis; (ii) a global asymptotical stable adaptive control scheme is presented for real systems; (iii) a sufficient condition of the closed-loop systems is given. Finally theoretical analysis verifies that the state converges to zero and all signals of the closed-loop systems are bounded.

\section{Problem Statement and Basic Assumptions}

Consider the nonlinear system in the following form:

$$
\begin{gathered}
\dot{x}_{i}=x_{i+1}, \quad i=1, \ldots, n-1, \\
\dot{x}_{n}=f(x)+g^{T}(x) u,
\end{gathered}
$$

where $x=\left(x_{1}, x_{2}, \ldots, x_{n}\right)^{T} \in R^{n}$ and $u \in R^{m}$ are the vectors of state and control input, respectively. $f(x)$ is the unknown continuous function; $g(x)$ is the vector of unknown continuous control gain function which satisfies $g_{j}(x) \geq g_{j \min }>0$, $j=1,2, \ldots, m$.

Definition 1 (see [19]). System (1) under the input being zero is globally asymptotically stable with decay rate $\alpha$, if there exists a scalar $\alpha>0$, such that

$$
\dot{V}(x(t)) \leq-2 \alpha V(x(t)),
$$

where $V(x(t))=x^{T}(t) P x(t)$ is the Lyapunov function candidate and $P>0$.

Lemma 2 (see [25]). Given two matrices $A$ and $B$ with appropriate dimensions, one has $A^{T B}+B^{T A} \leq A^{T A}+B^{T B}$.

In this paper, our objective is to design an adaptive fuzzy controller so that the closed-loop systems are asymptotically stable; that is, the states of the closed-loop system converge to zero and all signals of the closed-loop systems are bounded.

System (1) can be expressed in terms of the T-S fuzzy model as follows:

Plant rule $i$

IF $z_{1}(t)$ is $M_{1}^{i}, z_{2}(t)$ is $M_{2}^{i}, \ldots, z_{g}(t)$ is $M_{g}^{i}$

THEN $\dot{x}(t)=A_{i} x(t)+B_{i} u(t)+\left\{C_{i} u(t)\right\} x(t)+Q \Delta f_{i}(x, u)$

$i=1,2, \ldots, r$,

where $z(t)=\left[z_{1}(t), z_{2}(t), \ldots, z_{g}(t)\right] \in R^{g}$ are known premise variables that may be functions of the state variables, $M_{j}^{i}$ is the fuzzy set, $r$ is the number of the rules, $Q=[0, \ldots, 0,1]^{T}$, $\left\{C_{i} u(t)\right\}=\sum_{j=1}^{m} C_{i, j} u_{j}, C_{i, j} \in R^{n \times n}$ is constant matrices, and $\Delta f_{i}(x, u)=\left(\Delta a_{i} x+\Delta b_{i} u+\left\{\Delta C_{i} u\right\} x\right)$ denotes the model error of the $i$ th bilinear model in the $i$ th fuzzy space (also called the $i$ th fuzzy rule).

The modelling error terms are defined as follows:

$$
\begin{gathered}
\Delta a_{i}=\left(\Delta a_{i 1}, \Delta a_{i 2}, \ldots, \Delta a_{i n}\right) \in R^{1 \times n}, \\
\Delta b_{i}=\left(\Delta b_{i 1}, \Delta b_{i 2}, \ldots, \Delta b_{i m}\right) \in R^{1 \times m}, \\
\left\{\Delta C_{i} u(t)\right\}=\sum_{j=1}^{m} \Delta C_{i, j} u_{j}, \\
\Delta C_{i, j}=\left[\Delta c_{i, j 1}, \Delta c_{i, j 2} \cdots \Delta c_{i, j n}\right] \in R^{1 \times n} .
\end{gathered}
$$


$A_{i} \in R^{n \times n}, B_{i} \in R^{n \times m}$, and $C_{i, j}$ are constant matrices which have of the following forms:

$$
\begin{aligned}
A_{i} & =\left[\begin{array}{cccccc}
0 & 1 & 0 & \cdots & 0 & 0 \\
0 & 0 & 1 & \cdots & 0 & 0 \\
\vdots & \vdots & \vdots & & \vdots & \vdots \\
0 & 0 & 0 & \cdots & 1 & 0 \\
0 & 0 & 0 & \cdots & 0 & 1 \\
a_{i 1} & a_{i 2} & a_{i 3} & \cdots & a_{i(n-1)} & a_{i n}
\end{array}\right], \\
B_{i} & =\left[\begin{array}{cccccc}
0 & 0 & 0 & \cdots & 0 & 0 \\
0 & 0 & 0 & \cdots & 0 & 0 \\
\vdots & \vdots & \vdots & & \vdots & \vdots \\
0 & 0 & 0 & \cdots & 0 & 0 \\
0 & 0 & 0 & \cdots & 0 & 0 \\
b_{i 1} & b_{i 2} & b_{i 3} & \cdots & b_{i(m-1)} & b_{i m}
\end{array}\right], \\
C_{i, j} & =\left[\begin{array}{cccccc}
0 & 0 & 0 & \cdots & 0 & 0 \\
0 & 0 & 0 & \cdots & 0 & 0 \\
\vdots & \vdots & \vdots & & \vdots & \vdots \\
0 & 0 & 0 & \cdots & 0 & 0 \\
0 & 0 & 0 & \cdots & 0 & 0 \\
c_{i, j 1} & c_{i, j 2} & c_{i, j 3} & \cdots & c_{i, j(n-1)} & c_{i, j n}
\end{array}\right] .
\end{aligned}
$$

By using the fuzzy inference method with a singleton fuzzification, product inference, and centre average defuzzification, the overall fuzzy model is of the following form:

$$
\begin{gathered}
\dot{x}(t)=\sum_{i=1}^{r} h_{i}(z(t))\left[A_{i} x(t)+B_{i} u(t)+\left\{C_{i} u(t)\right\} x(t)\right. \\
\left.+Q \Delta f_{i}(x, u)\right]
\end{gathered}
$$

where

$$
\begin{aligned}
& h_{i}(z(t))=\frac{w_{i}(z(t))}{\sum_{j=1}^{r} w_{j}(z(t))}, \\
& w_{i}(z(t))=\prod_{j=1}^{g} M_{j}^{i}\left(z_{j}(t)\right) .
\end{aligned}
$$

We assume $w_{i}(z(t)) \geq 0, \sum_{i=1}^{r} w_{i}(z(t))>0$, for all $t$. Therefore, we have

$$
h_{i}(z(t)) \geq 0, \quad \sum_{i=1}^{r} h_{i}(z(t))=1, \quad i=1,2, \ldots, r .
$$

By comparison with (1) and (6), it is easy to see that

$$
\begin{gathered}
f(x)=\sum_{i=1}^{r} h_{i}(z(t))\left(a_{i}+\Delta a_{i}\right) x(t), \\
g_{j}(x)=\sum_{i=1}^{r} h_{i}(z(t))\left(b_{i, j}+\Delta b_{i, j}+c_{i, j} x(t)+\Delta C_{i, j} x(t)\right),
\end{gathered}
$$

where $a_{i}=\left(a_{i 1}, a_{i 2}, \ldots, a_{i n}\right) \in R^{1 \times n}, b_{i}=\left(b_{i 1}, b_{i 2}, \ldots, b_{i m}\right) \in$ $R^{1 \times m}$, and $c_{i, j}=\left(c_{i, j 1}, c_{i, j 2}, \ldots, c_{i, j n}\right)$.
Remark 3. From now on, unless confusion arises, arguments such as $z(t)$ in $h_{i}(z(t))$ will be omitted just for notational convenience.

\section{Control Design and Stability Analysis}

System (6) can be represented by following the T-S fuzzy model without considering the modelling error; that is, $\Delta f_{i}(x, u) \equiv 0, i=1,2, \ldots, r$. Consider

Plant rule $i$

$$
\begin{aligned}
& \text { IF } z_{1}(t) \text { is } M_{1}^{i}, z_{2}(t) \text { is } M_{2}^{i}, \ldots, z_{g}(t) \text { is } M_{g}^{i} \\
& \text { THEN } \dot{x}(t)=A_{i} x(t)+B_{i} u(t)+\left\{C_{i} u(t)\right\} x(t) \\
& i=1,2, \ldots, r .
\end{aligned}
$$

By using the fuzzy inference method with a singleton fuzzification, product inference, and centre average defuzzification, the overall fuzzy model is of the following form:

$$
\dot{x}(t)=\sum_{i=1}^{r} h_{i}\left[A_{i} x(t)+B_{i} u(t)+\left\{C_{i} u(t)\right\} x(t)\right] .
$$

Based on the idea of PDC, the $j$ th state-feedback controller is designed as follows:

Plant rule $i$

IF $z_{1}(t)$ is $M_{1}^{i}, z_{2}(t)$ is $M_{2}^{i}, \ldots, z_{g}(t)$ is $M_{g}^{i}$

$$
\operatorname{THEN} u_{q, j}=\frac{\rho_{j} F_{i, j} x(t)}{\sqrt{1+x^{T}(t) F_{i, j}^{T} F_{i, j} x(t)}},
$$

$$
i=1,2, \ldots, r \text {, }
$$

where $F_{i, j} \in R^{1 \times n}$ is a vector to be determined and $\rho_{j}>0$ is a scalar to be assigned.

The overall fuzzy control law can be represented by

$$
\begin{aligned}
u_{q, j} & =\sum_{i=1}^{r} h_{i} \frac{\rho_{j} F_{i, j} x(t)}{\sqrt{1+x^{T}(t) F_{i, j}^{T} F_{i, j} x(t)}} \\
& =\sum_{i=1}^{r} h_{i} \rho_{j} \sin \theta_{i, j}=\sum_{i=1}^{r} h_{i} \rho_{j} F_{i, j} \cos \theta_{i, j} x(t),
\end{aligned}
$$

where

$$
\begin{array}{r}
\sin \theta_{i, j}=\frac{F_{i, j} x(t)}{\sqrt{1+x^{T}(t) F_{i, j}^{T} F_{i, j} x(t)}}, \\
\cos \theta_{i, j}=\frac{1}{\sqrt{1+x^{T}(t) F_{i, j}^{T} F_{i, j} x(t)}}, \\
\theta_{i, j} \in\left[-\frac{\pi}{2}, \frac{\pi}{2}\right], \\
1 \leq i \leq r, \quad 1 \leq j \leq m .
\end{array}
$$


Substituting (13) into (11), one can get the closed-loop system

$$
\begin{array}{r}
\dot{x}(t)=\sum_{i=1}^{r} \sum_{j=1}^{r} h_{i} h_{j}\left(A_{i}+\sum_{k=1}^{m} B_{i, k} \rho_{k} F_{j, k} \cos \theta_{j, k}\right. \\
\left.+\sum_{k=1}^{m} C_{i, k} \rho_{k} \sin \theta_{j, k}\right) x(t),
\end{array}
$$

where $B_{i, k}$ denotes the $k$ th column of the $B_{i}$.

Theorem 4. Given positive scalars $\rho_{k}(1 \leq k \leq m)$, if there exist a symmetric positive definite matrix $U$ and some constant matrices $W_{i, j}$, such that LMIs (16) and (17) hold,

$$
\begin{aligned}
& {\left[\begin{array}{ccc}
A_{i} U+U A_{i}^{T}+\bar{\rho}+2 \alpha U & * & * \\
\bar{B}_{i} \bar{W}_{i} & -\bar{I} & \\
\bar{C}_{i} U & & -\bar{I}
\end{array}\right]<0, \quad 1 \leq i \leq r,} \\
& {\left[\begin{array}{lllll}
M_{i} & * & * & * & * \\
\bar{B}_{i} \bar{W}_{j} & -\bar{I} & & & \\
\bar{B}_{j} \bar{W}_{i} & & -\bar{I} & & \\
\bar{C}_{i} U & & & -\bar{I} & \\
\bar{C}_{j} U & & & & -\bar{I}
\end{array}\right]<0, \quad 1 \leq i<j \leq r,}
\end{aligned}
$$

where $\bar{\rho}=\sum_{k=1}^{m} \rho_{k}^{2}, M_{i}=A_{i} U+U A_{i}^{T}+A_{j} U+U A_{j}^{T}+2 \bar{\rho}+2 \alpha U$,

$$
\begin{gathered}
\bar{B}_{i} \bar{W}_{i}=\left[\begin{array}{c}
B_{i, 1} W_{i, 1} \\
\vdots \\
B_{i, m} W_{i, m}
\end{array}\right], \quad \bar{C}_{i}=\left[\begin{array}{c}
C_{i, 1} \\
\vdots \\
C_{i, m}
\end{array}\right], \\
\bar{I}=\left[\begin{array}{lll}
I & & \\
& \ddots & \\
& & I
\end{array}\right] .
\end{gathered}
$$

Then, the FBS (15) is globally asymptotically stable with decay rate $\alpha$ via the fuzzy feedback controller (13), and the gains can be determined by $F_{i, j}=W_{i, j} U^{-1}$.

Proof. Consider the Lyapunov function candidate as follows:

$$
V_{1}(x(t))=x^{T}(t) P x(t)
$$

where $P=U^{-1}$.

Applying Schur complement lemma, inequality (16) can be written as follows:

$$
\begin{gathered}
U A_{i}^{T}+A_{i} U+\sum_{k=1}^{m} \rho_{k}^{2}+\sum_{k=1}^{m}\left(B_{i, k} W_{i, k}\right)^{T} B_{i, k} W_{i, k} \\
+\sum_{k=1}^{m}\left(C_{i, k} U\right)^{T}\left(C_{i, k} U\right)+2 \alpha U<0 .
\end{gathered}
$$

Premultiplying and postmultiplying (20) by $P$, respectively, we have

$$
\begin{aligned}
& A_{i}^{T} P+P A_{i}+\sum_{k=1}^{m} \rho_{k}^{2} P P+\sum_{k=1}^{m}\left(B_{i, k} F_{i, k}\right)^{T} B_{i, k} F_{i, k} \\
& +\sum_{k=1}^{m} C_{i, k}^{T} C_{i, k}+2 \alpha P<0 .
\end{aligned}
$$

Applying a similar procedure to inequality (17), we can obtain

$$
\begin{aligned}
& A_{i}^{T} P+P A_{i}+A_{j}^{T} P+P A_{j}+2 \sum_{k=1}^{m} \rho_{k}^{2} P P \\
& \quad+\sum_{k=1}^{m}\left(B_{i, k} F_{j, k}\right)^{T}\left(B_{i, k} F_{j, k}\right)+\sum_{k=1}^{m} C_{i, k}^{T} C_{i, k} \\
& \quad+\sum_{k=1}^{m}\left(B_{j, k} F_{i, k}\right)^{T}\left(B_{j, k} F_{i, k}\right)+\sum_{k=1}^{m} C_{j, k}^{T} C_{j, k}+2 \alpha P<0 .
\end{aligned}
$$

The time derivative of $V_{1}$ is

$$
\dot{V}_{1}(x(t))=\dot{x}^{T} P x+x^{T} P \dot{x} .
$$

By substituting (15) into (23), we can get

$$
\begin{gathered}
\dot{V}_{1}(x(t))=\sum_{i=1}^{r} \sum_{j=1}^{r} h_{i} h_{j} x^{T}\left\{\left[A_{i}+\sum_{k=1}^{m} B_{i, k} \rho_{k} F_{j, k} \cos \theta_{j, k}\right.\right. \\
\left.+\sum_{k=1}^{m} C_{i, k} \rho_{k} \sin \theta_{j, k}\right]^{T} P \\
+P\left[A_{i}+\sum_{k=1}^{m} B_{i, k} \rho_{k}\right. \\
\times F_{j, k} \cos \theta_{j, k} \\
\left.\left.+\sum_{k=1}^{m} C_{i, k} \rho_{k} \sin \theta_{j, k}\right]\right\} \\
=\sum_{i=1}^{r} h_{i}^{2} x^{T} \Lambda_{i, i} x+\sum_{i<j}^{r} h_{i} h_{j} x^{T} \Lambda_{i, j} x,
\end{gathered}
$$


where

$$
\begin{aligned}
\Lambda_{i, i}= & A_{i}^{T} P+P A_{i}+\sum_{k=1}^{m} \rho_{k} \sin \theta_{i, k}\left(C_{i, k}^{T} P+P C_{i, k}\right) \\
& +\sum_{k=1}^{m} \rho_{k} \cos \theta_{i, k}\left[\left(B_{i, k} F_{i, k}\right)^{T} P+P B_{i, k} F_{i, k}\right], \\
\Lambda_{i, j}= & A_{i}^{T} P+P A_{i}+\sum_{k=1}^{m} \rho_{k} \sin \theta_{i, k}\left(C_{i, k}^{T} P+P C_{i, k}\right) \\
& +\sum_{k=1}^{m} \rho_{k} \cos \theta_{i, k}\left[\left(B_{i, k} F_{j, k}\right)^{T} P+P B_{i, k} F_{j, k}\right] \\
& +A_{j}^{T} P+P A_{j}+\sum_{k=1}^{m} \rho_{k} \sin \theta_{j, k}\left(C_{j, k}^{T} P+P C_{j, k}\right) \\
& +\sum_{k=1}^{m} \rho_{k} \cos \theta_{j, k}\left[\left(B_{j, k} F_{i, k}\right)^{T} P+P B_{j, k} F_{i, k}\right] .
\end{aligned}
$$

First, by premultiplying and postmultiplying $\Lambda_{i, i}$ by $U$, we can obtain

$$
\begin{aligned}
U \Lambda_{i, i} U= & U A_{i}^{T}+A_{i} U+\sum_{k=1}^{m} \rho_{k} \sin \theta_{i, k}\left(U C_{i, k}^{T}+C_{i, k} U\right) \\
& +\sum_{k=1}^{m} \rho_{k} \cos \theta_{i, k}\left[U\left(B_{i, k} F_{i, k}\right)^{T}+B_{i, k} F_{i, k} U\right] .
\end{aligned}
$$

According to Lemma 2, we can get the following:

$$
\begin{gathered}
{\left[\rho_{k} B_{i, k} F_{i, k} U+\rho_{k} U\left(B_{i, k} F_{i, k}\right)^{T}\right] \cos \theta_{i, k}} \\
\leq \rho_{k}^{2} \cos ^{2} \theta_{i, k}+\left(B_{i, k} W_{i, k}\right)^{T} B_{i, k} W_{i, k}, \\
U C_{i, k}^{T} \rho_{k} \sin \theta_{i, k}+C_{i, k} U \rho_{k} \sin \theta_{i, k} \\
\leq \rho_{k}^{2} \sin ^{2} \theta_{i, k}+\left(C_{i, k} U\right)^{T}\left(C_{i, k} U\right) .
\end{gathered}
$$

From (26) and (27), we can obtain

$$
\begin{aligned}
U \Lambda_{i, i} U \leq & U A_{i}^{T}+A_{i} U+\sum_{k=1}^{m} \rho_{k}^{2}+\sum_{k=1}^{m}\left(C_{i, k} U\right)^{T} C_{i, k} U \\
& +\sum_{k=1}^{m}\left(B_{i, k} W_{i, k}\right)^{T} B_{i, k} W_{i, k} .
\end{aligned}
$$

Applying similar procedures (26)-(28) to $\Lambda_{i, j}$, we can obtain

$$
\begin{aligned}
U \Lambda_{i, j} U \leq & U A_{i}^{T}+A_{i} U+U A_{j}^{T}+A_{j} U+2 \sum_{k=1}^{m} \rho_{k}^{2} \\
& +\sum_{k=1}^{m}\left(B_{i, k} W_{j, k}\right)^{T}\left(B_{i, k} W_{j, k}\right)+\sum_{k=1}^{m}\left(C_{i, k} U\right)^{T} C_{i, k} U \\
& +\sum_{k=1}^{m}\left(B_{j, k} W_{i, k}\right)^{T}\left(B_{j, k} W_{i, k}\right)+\sum_{k=1}^{m}\left(C_{j, k} U\right)^{T} C_{j, k} U .
\end{aligned}
$$

Substituting (28) and (29) into (24), we obtain

$$
\begin{aligned}
& \dot{V}_{1}(x(t)) \leq \sum_{i=1}^{r} h_{i}^{2} x^{T} P\left[U A_{i}^{T}\right.+A_{i} U+\sum_{k=1}^{m} \rho_{k}^{2} \\
&+\sum_{k=1}^{m}\left(B_{i, k} W_{i, k}\right)^{T} B_{i, k} W_{i, k} \\
&+\left.\sum_{k=1}^{m}\left(C_{i, k} U\right)^{T}\left(C_{i, k} U\right)\right] P x \\
&+\sum_{i<j}^{r} h_{i} h_{j} x^{T} P\left[U A_{i}^{T}+A_{i} U+U A_{j}^{T}\right. \\
&+A_{j} U+2 \sum_{k=1}^{m} \rho_{k}^{2} \\
&+\sum_{k=1}^{m}\left(B_{i, k} W_{j, k}\right)^{T}\left(B_{i, k} W_{j, k}\right) \\
&+\sum_{k=1}^{m}\left(C_{i, k} U\right)^{T} C_{i, k} U \\
&+\sum_{k=1}^{m}\left(B_{j, k} W_{i, k}\right)^{T}\left(B_{j, k} W_{i, k}\right) \\
&\left.+\sum_{k=1}^{m}\left(C_{j, k} U\right)^{T} C_{j, k} U\right] P x .
\end{aligned}
$$

By substituting (21) and (22) into (30), we can obtain

$$
\dot{V}_{1}(x(t)) \leq-2 \alpha P x=-2 \alpha V_{1}(x(t)) .
$$

Then, by Definition 1, the closed-loop fuzzy system (15) is globally asymptotically stable with decay rate $\alpha$. This completes the proof of Theorem 4.

Next, the modelling error in (6) is considered and an adaptive compensation term is adopted to reduce the effects of the modelling error.

Adopt the fuzzy controller in the following form:

$$
u=u_{q, j}+u_{s, j}, \quad j=1,2, \ldots, m,
$$

where compensator $u_{s, j}$ will be designed later.

Substituting (32) into (6) yields

$$
\begin{aligned}
\dot{x}(t)=\sum_{i=1}^{r} \sum_{j=1}^{r} h_{i} h_{j}\left[\left(A_{i}\right.\right. & +\sum_{k=1}^{m} B_{i, k} \rho_{k} F_{j, k} \cos \theta_{j, k} \\
& \left.+\sum_{k=1}^{m} C_{i, k} \rho_{k} \sin \theta_{j, k}\right) x(t) \\
+ & Q\left(\Delta a_{i}+\sum_{k=1}^{m} \Delta b_{i, k} \rho_{k} F_{j, k} \cos \theta_{j, k}\right. \\
& \left.+\sum_{k=1}^{m} \Delta C_{i, k} \rho_{k} \sin \theta_{j, k}\right) x(t)
\end{aligned}
$$




$$
\begin{array}{r}
+\sum_{k=1}^{m}\left(B_{i, k}+Q \Delta b_{i, k}+C_{i, k} x(t)\right. \\
\left.\left.+Q \Delta C_{i, k} x(t)\right) u_{s, j}\right] .
\end{array}
$$

Suppose that there exists an unknown constant $\lambda$ such that

$$
\lambda \geq \sum_{i=1}^{r} \sum_{j=1}^{r}\left\|\Delta a_{i}+\sum_{k=1}^{m} \Delta b_{i, k} \rho_{k} F_{j, k} \cos \theta_{j, k}+\sum_{k=1}^{m} \Delta C_{i, k} \rho_{k} \sin \theta_{j, k}\right\| .
$$

Then, from $0 \leq h_{i}(z(t)) \leq 1$ and

$$
\begin{gathered}
\left\|\sum_{i=1}^{r} \sum_{j=1}^{r} h_{i} h_{j}\left(\Delta a_{i}+\sum_{k=1}^{m} \Delta b_{i, k} \rho_{k} F_{j, k} \cos \theta_{j, k}+\sum_{k=1}^{m} \Delta C_{i, k} \rho \sin \theta_{j, k}\right)\right\| \\
\leq \sum_{i=1}^{r} \sum_{j=1}^{r}\left\|\Delta a_{i}+\sum_{k=1}^{m} \Delta b_{i, k} \rho_{k} F_{j, k} \cos \theta_{j, k}+\sum_{k=1}^{m} \Delta C_{i, k} \rho \sin \theta_{j, k}\right\|
\end{gathered}
$$

we have

$$
\begin{array}{r}
\| \sum_{i=1}^{r} \sum_{j=1}^{r} h_{i} h_{j}\left(\Delta a_{i}+\sum_{k=1}^{m} \Delta b_{i, k} \rho_{k} F_{j, k} \cos \theta_{j, k}\right. \\
\left.+\sum_{k=1}^{m} \Delta C_{i, k} \rho \sin \theta_{j, k}\right) \| \leq \lambda .
\end{array}
$$

It is easy to see that we can choose a function vector $H_{\lambda}$ such that

$$
\begin{aligned}
\sum_{i=1}^{r} \sum_{j=1}^{r} h_{i} h_{j}\left(\Delta a_{i}\right. & +\sum_{k=1}^{m} \Delta b_{i, k} \rho_{k} F_{j, k} \cos \theta_{j, k} \\
& \left.+\sum_{k=1}^{m} \Delta C_{i, k} \rho_{k} \sin \theta_{j, k}\right)=\lambda H_{\lambda}
\end{aligned}
$$

and $\left\|H_{\lambda}\right\| \leq 1$.

Remark 5. Here assumption (34) is reasonable in many real systems due to its boundedness, such as chaotic system [19]; for example, Example 1 in the paper satisfies the assumption. On the other hand, the uncertain terms of the considered systems in the existing literature [2, 18, 22-24] satisfy the condition of (34).

Denote

$$
\omega(t)=H_{\lambda} x(t)
$$

Substituting (37) and (38) into (33), we can obtain the following feedback system:

$$
\begin{aligned}
\dot{x}(t)=\sum_{i=1}^{r} \sum_{j=1}^{r} h_{i} h_{j}\left[\left(A_{i}+\sum_{k=1}^{m} B_{i, k} \rho_{k} F_{j, k} \cos \theta_{j, k}\right.\right. \\
\left.+\sum_{k=1}^{m} C_{i, k} \rho_{k} \sin \theta_{j, k}\right) x(t) \\
+\sum_{k=1}^{m}\left(B_{i, k}+Q \Delta b_{i, k}+C_{i, k} x(t)\right. \\
\left.\left.+Q \Delta C_{i, k} x(t)\right) u_{s, j}\right]+Q \lambda \omega(t) .
\end{aligned}
$$

Choose the adaptive compensator as follows:

$$
u_{s, j}=-\frac{1}{2 m g_{j \min } \gamma^{2}} Q^{T} P \widehat{c} x(t), \quad j=1,2, \ldots, m,
$$

where $c=\lambda^{2}, \widehat{c}$ is the parameter estimation of $c$, and $\gamma>0$ is a gain constant.

Choose the adaptive law as follows:

$$
\dot{\hat{c}}=\frac{1}{2 \eta \gamma^{2}} x^{T}(t) P Q Q^{T} P x(t),
$$

where $\eta>0$ is a gain constant which determines the rate of adaptation.

Substituting (40) into (39) yields

$$
\begin{aligned}
\dot{x}(t)=\sum_{i=1}^{r} \sum_{j=1}^{r} h_{i} h_{j}\left[\left(A_{i}+\sum_{k=1}^{m} B_{i, k} \rho_{k} F_{j, k} \cos \theta_{j, k}\right.\right. \\
\left.+\sum_{k=1}^{m} C_{i, k} \rho_{k} \sin \theta_{j, k}\right) x(t) \\
+\sum_{k=1}^{m}\left(B_{i, k}+Q \Delta b_{i, k}+C_{i, k} x(t)\right. \\
\left.\times\left(-\frac{Q^{T} P \widehat{c} x(t)}{2 m g_{j \min } \gamma^{2}}\right)\right]+Q \lambda \omega(t) .
\end{aligned}
$$

Theorem 6. Consider the uncertain nonlinear system (1) with control law defined by (32), (13), and (40) and the parameter updated by the adaptive law (41). If there exist a symmetric positive definite matrix $P$ and some matrices $F_{i, j}(1 \leq i, j \leq r)$ satisfying the LMIs (16) and (17) and the design parameter is chosen as

$$
0<\gamma<\sqrt{2 \alpha \lambda_{\min }(P)}
$$

then the closed-loop system (42) is asymptotically stable and all signals of the closed-loop system (42) are bounded. 
Proof. Consider the Lyapunov function candidate

$$
V=x^{T}(t) P x(t)+\eta(\widehat{c}-c)^{2},
$$

where $\eta>0$.

Let

$$
G_{i, j}=A_{i}+\sum_{k=1}^{m} B_{i, k} \rho_{k} F_{j, k} \cos \theta_{j, k}+\sum_{k=1}^{m} C_{i, k} \rho_{k} \sin \theta_{j, k} .
$$

The time derivative of $V$ is

$$
\dot{V}=\dot{x}^{T} P x+x^{T} P \dot{x}+2 \eta(\widehat{c}-c) \dot{\vec{c}} .
$$

Substituting (39) into (46), we can obtain

$$
\begin{aligned}
& \dot{V}=\sum_{i=1}^{r} \sum_{j=1}^{r} h_{i} h_{j}\left[x^{T}(t)\right. \\
& \times\left\{G_{i, j}{ }^{T} P+P G_{i, j}+2 P Q\right. \\
& \times\left(\Delta a_{i}+\sum_{k=1}^{m} \Delta b_{i, k} \rho_{k} F_{j, k} \cos \theta_{j, k}\right. \\
& \left.\left.+\sum_{k=1}^{m} \Delta C_{i, k} \rho_{k} \sin \theta_{j, k}\right)\right\} x(t) \\
& +2 x^{T}(t) P \sum_{k=1}^{m}\left(B_{i, k}+Q \Delta b_{i, k}+C_{i, k} x(t)\right. \\
& \left.\left.+Q \Delta C_{i, k} x(t)\right) u_{s, k}\right] \\
& +2 \eta(\widehat{c}-c) \dot{\widehat{c}} \\
& =\sum_{i=1}^{r} \sum_{j=1}^{r} h_{i} h_{j} x^{T}(t)\left(G_{i, j}{ }^{T} P+P G_{i, j}\right) x(t) \\
& +2 x^{T}(t) P Q \lambda \omega(t) \\
& +2 x^{T}(t) P Q \sum_{j=1}^{m} g_{j}(x) u_{s, j}+2 \eta(\widehat{c}-c) \dot{\hat{c}} .
\end{aligned}
$$

From the proof of Theorem 4, we get

$$
\begin{aligned}
\dot{V} \leq & -2 \alpha x^{T}(t) P x(t)+2 x^{T}(t) P Q \lambda \omega(t) \\
& +2 x^{T}(t) P Q \sum_{j=1}^{m} g_{j}(x) u_{s, j}+2 \eta(\widehat{c}-c) \dot{\hat{c}} .
\end{aligned}
$$

It is easy to see that

$$
\begin{aligned}
& 2 x^{T} P Q \lambda \omega-\gamma^{2}\|\omega\|^{2}+\gamma^{2}\|\omega\|^{2} \\
& =-\gamma^{2}\left\|\omega-\frac{1}{\gamma^{2}} x^{T} P Q \lambda\right\|^{2}+\frac{1}{\gamma^{2}} x^{T} P Q \lambda^{2} Q^{T} P x+\gamma^{2}\|\omega\|^{2} \\
& \leq \frac{\lambda^{2}}{\gamma^{2}} x^{T} P Q Q^{T} P x+\gamma^{2}\|\omega\|^{2} .
\end{aligned}
$$

Substituting (49) into (48) yields

$$
\begin{aligned}
\dot{V} \leq & -2 \alpha x^{T}(t) P x(t)+\frac{\lambda^{2}}{\gamma^{2}} x^{T} P Q Q^{T} P x+\gamma^{2}\|\omega\|^{2} \\
& +2 x^{T}(t) P Q \sum_{j=1}^{m} g_{j}(x) u_{s, j}+2 \eta(\widehat{c}-c) \dot{\hat{c}} .
\end{aligned}
$$

Substituting (40) and (41) into (50), we obtain

$$
\begin{aligned}
\dot{V} \leq & -2 \alpha x^{T}(t) P x(t)+(-\widehat{c}+c) \frac{1}{\gamma^{2}} x^{T} P Q Q^{T} P x \\
& +2 \eta(\widehat{c}-c) \dot{\hat{c}}+\gamma^{2}\|\omega\|^{2} \\
= & -2 \alpha x^{T}(t) P x(t)+\gamma^{2}\|\omega\|^{2} \\
\leq & -2 \alpha \lambda_{\min }(P)\|x(t)\|^{2}+\gamma^{2}\|\omega\|^{2},
\end{aligned}
$$

where $\|\omega\|^{2}=\omega^{T} \omega=x^{T} H_{\lambda}{ }^{T} H_{\lambda} x \leq\left\|H_{\lambda}{ }^{T} H_{\lambda}\right\| \cdot\|x\|^{2} \leq\|x\|^{2}$.

By choosing $0<\gamma<\sqrt{2 \alpha \lambda_{\min }(P)}$, we can get $\dot{V}<0$; then, we have that the states $x(t) \rightarrow 0$ as $t$ approaches infinity via LaSalle invariance principle and $V(t)$ is bounded. From (44), we can obtain that states $x$ and $\widehat{c}$ are bounded; therefore, the boundedness of $u_{s, j}$ is ensured from (40). Similarly, from (13), we can obtain that $u_{q, j}$ is bounded. Then it can be proved that (1) the closed-loop system (42) is asymptotically stable and (2) all signals of the closed-loop system (42) are bounded.

\section{Simulations}

In this section, we will give two examples to show the efficiency of the proposed approach. The first example is an unknown chaotic system, and the second example is a parameter uncertain T-S fuzzy bilinear system with multiple inputs.

Example 1. Consider the following chaotic system with control input:

$$
\begin{gathered}
\dot{x}_{1}=x_{2}, \\
\dot{x}_{2}=-0.1 x_{2}-x_{1}^{3}+12 \cos t+u .
\end{gathered}
$$

When $u(t)=0$ and the initial states are chosen as $x(0)=$ $(2,2)^{T}$, the states phase portrait of system (52) is shown in Figure 1. 


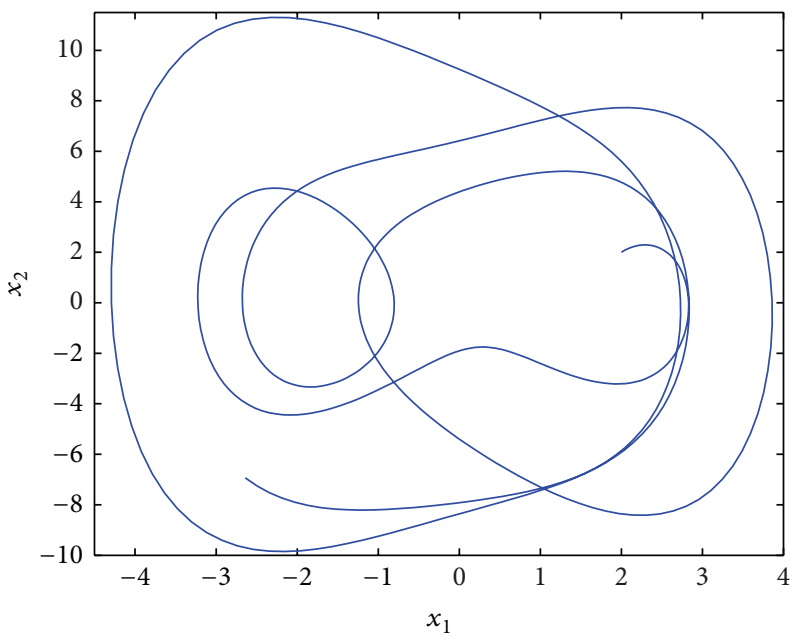

FIGURE 1: The phase portrait of the chaotic system.

System (52) can be modelled as the following T-S fuzzy bilinear model:

Rule 1 IF $x_{1}(t)$ is about 0

$$
\begin{aligned}
\text { THEN } \dot{x}(t)= & \left(A_{1}+\Delta A_{1}\right) x(t)+\left(B_{1}+\Delta B_{1}\right) u(t) \\
& +\left(C_{1}+\Delta C_{1}\right) x(t) u(t) ;
\end{aligned}
$$

Rule 2 IF $x_{1}(t)$ is about \pm 2

$$
\begin{aligned}
\text { THEN } \dot{x}(t)= & \left(A_{2}+\Delta A_{2}\right) x(t)+\left(B_{2}+\Delta B_{2}\right) u(t) \\
& +\left(C_{2}+\Delta C_{2}\right) x(t) u(t) ;
\end{aligned}
$$

Rule 3 IF $x_{1}(t)$ is about \pm 4

$$
\begin{aligned}
\text { THEN } \dot{x}(t)= & \left(A_{3}+\Delta A_{3}\right) x(t)+\left(B_{3}+\Delta B_{3}\right) u(t) \\
& +\left(C_{3}+\Delta C_{3}\right) x(t) u(t),
\end{aligned}
$$

where $x(t)=\left(x_{1}(t), x_{2}(t)\right)^{T}, A_{1}=A_{2}=A_{3}=\left[\begin{array}{cc}0 & 1 \\ -1 & -1\end{array}\right], B_{1}=$ $B_{2}=B_{3}=\left[\begin{array}{c}0 \\ -1\end{array}\right]$, and $C_{1}=C_{2}=C_{3}=\left[\begin{array}{ll}0 & 1 \\ 1 & 1\end{array}\right]$. Choose $\alpha=0.3$, $\rho=0.09, F_{1}=\left[\begin{array}{ll}0 & -1\end{array}\right], F_{2}=\left[\begin{array}{ll}-1 & -1\end{array}\right]$, and $F_{3}=\left[\begin{array}{ll}-1 & -1\end{array}\right]$. By solving LMIs (16)-(17), one can obtain

$$
P=\left[\begin{array}{cc}
9.3637 & 4.6993 \\
4.6993 & 10.6246
\end{array}\right], \quad \lambda_{\text {min }}(P)=5.2528 .
$$

Utilize the controllers (32), (13), and (40) and the parameter updated law (41) to control system (52). The design parameters are chosen as $\eta=2, \gamma=2<\sqrt{\lambda_{\min }(P)}$, the initial conditions are chosen as $x(0)=(2,-2)^{T}, \widehat{c}(0)=0$, and the relationship functions are selected as shown in Figure 2. The simulation results are shown in Figures 3, 4, 5, and 6. In Figures 3-6, the curves of states, control input, and adaptive updated parameter for the T-S fuzzy bilinear system are drawn by solid lines, respectively, while the curves of states, control input, and adaptive updated parameter for T-S fuzzy linear system are depicted by dotted lines, respectively. By comparison,

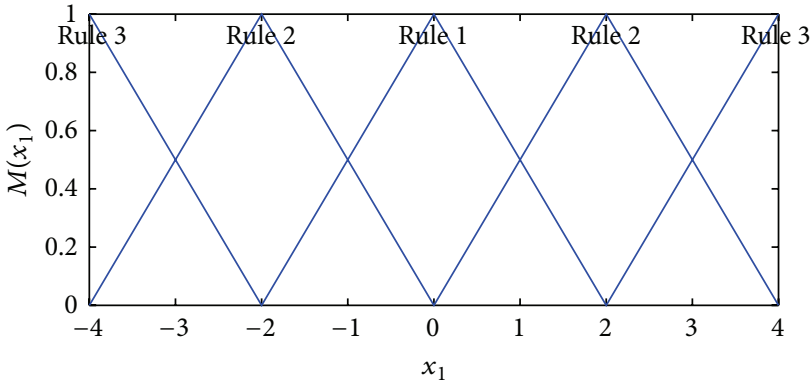

FIGURE 2: The relationship functions.

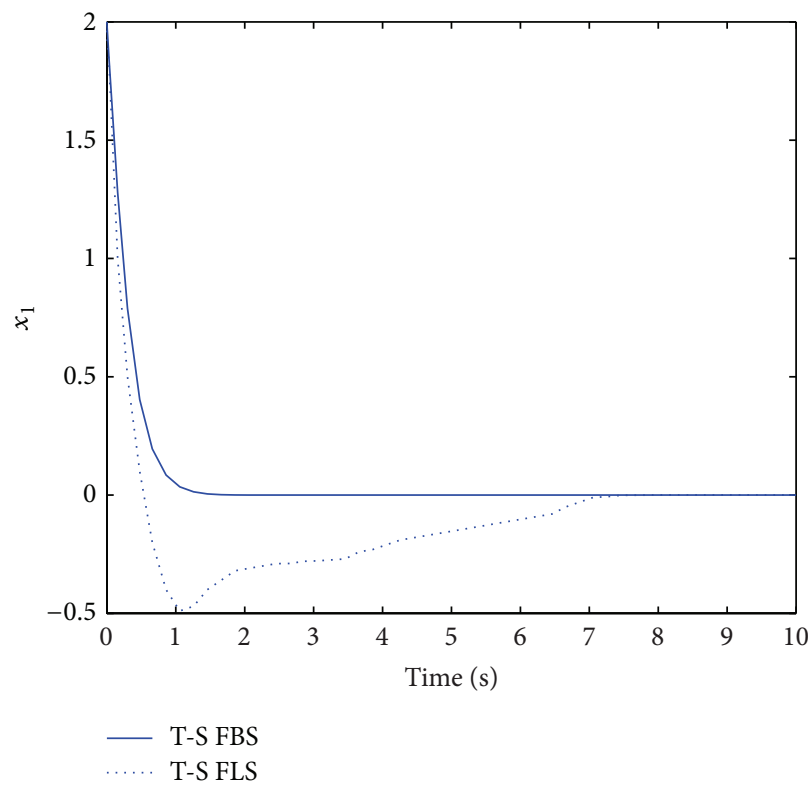

FiguRE 3: The state $x_{1}$ response curves.

the convergence rates of the states of two systems are almost the same, though the state and control amplitudes of T-S fuzzy bilinear system (FBS) are smaller than T-S fuzzy linear system (FLS). Thus, the proposed method has some advantages of performance over the existing approach [16].

Example 2. Consider the following parametric uncertain multiple inputs bilinear fuzzy system:

Rule 1 IF $x_{1}(t)$ is $L_{1}$

$$
\begin{aligned}
\text { THEN: } \dot{x}(t)= & A_{1} x(t)+B_{1} u(t)+\left\{C_{1} u(t)\right\} x(t) \\
+ & Q\left(\Delta a_{1} x(t)+\Delta b_{1} u(t)\right. \\
& \left.+\left\{\Delta C_{1} u(t)\right\} x(t)\right) ;
\end{aligned}
$$

Rule 2 IF $x_{2}(t)$ is $L_{2}$

$$
\begin{aligned}
\text { THEN: } \dot{x}(t)= & A_{2} x(t)+B_{2} u(t)+\left\{C_{2} u(t)\right\} x(t) \\
+ & Q\left(\Delta a_{2} x(t)+\Delta b_{2} u(t)\right. \\
& \left.+\left\{\Delta C_{2} u(t)\right\} x(t)\right),
\end{aligned}
$$




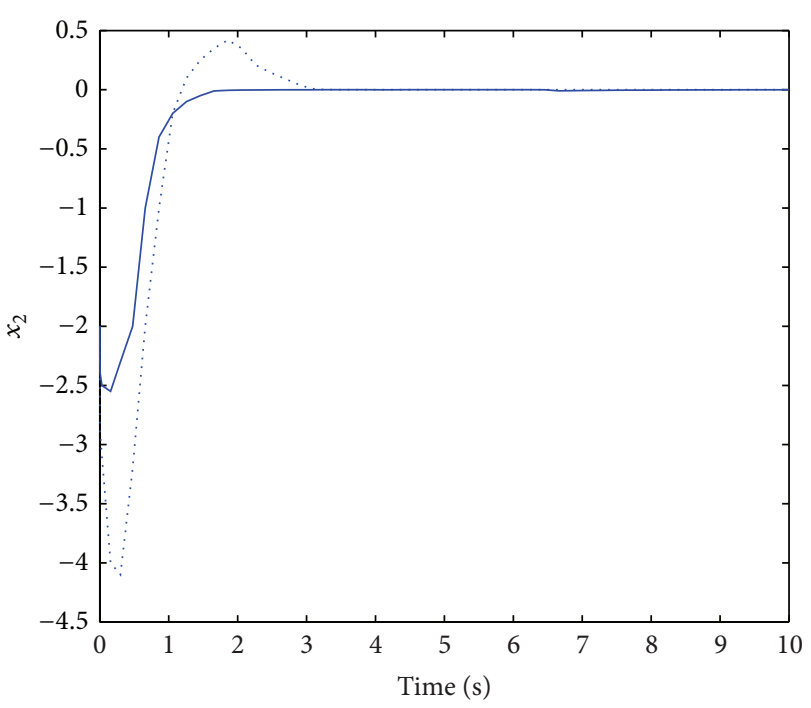

T-S FLS

- T-S FBS

FIgURE 4: The state $x_{2}$ response curves.

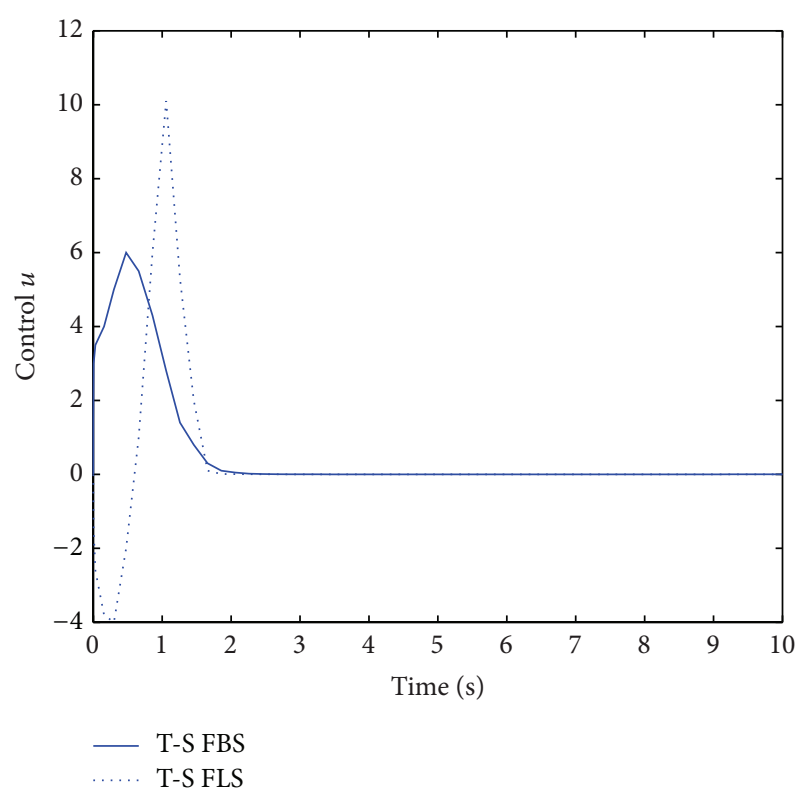

FIGURE 5: The control curves.

where $x(t)=\left(x_{1}(t), x_{2}(t)\right)^{T}, A_{1}=A_{2}=\left[\begin{array}{cc}0 & 1 \\ -1 & -1\end{array}\right], B_{1,1}=\left[\begin{array}{l}0 \\ 1\end{array}\right]$, $B_{1,2}=\left[\begin{array}{c}0 \\ -1\end{array}\right], B_{2,1}=B_{2,2}=\left[\begin{array}{l}0 \\ 1\end{array}\right], C_{1,1}=\left[\begin{array}{cc}0 & 0 \\ -1 & 1\end{array}\right], C_{1,2}=C_{2,1}=$ $C_{2,2}=\left[\begin{array}{cc}0 & 0 \\ -1 & -1\end{array}\right], \alpha=0.3, \rho_{1}=0.05, \rho_{2}=0.04, F_{1,1}=$ $\left[\begin{array}{ll}-1.2 & -1.8\end{array}\right], F_{1,2}=\left[\begin{array}{ll}-0.8 & -0.9\end{array}\right], F_{2,1}=\left[\begin{array}{ll}-0.5 & -1.2\end{array}\right]$, and $F_{2,2}=\left[\begin{array}{ll}-1 & -1\end{array}\right]$; using LMI technique to solve (16)-(17), we can get a feasible solution as

$$
P=\left[\begin{array}{cc}
16.8099 & 8.5379 \\
8.5379 & 18.0100
\end{array}\right], \quad \lambda_{\min }(P)=8.8510 .
$$

Apply the controllers (32), (13), and (40) and the parameters updated law (41) to system (55). The design parameters are chosen as $\eta=2, \gamma=2<\sqrt{2 \alpha \lambda_{\min }(P)}$. The initial conditions

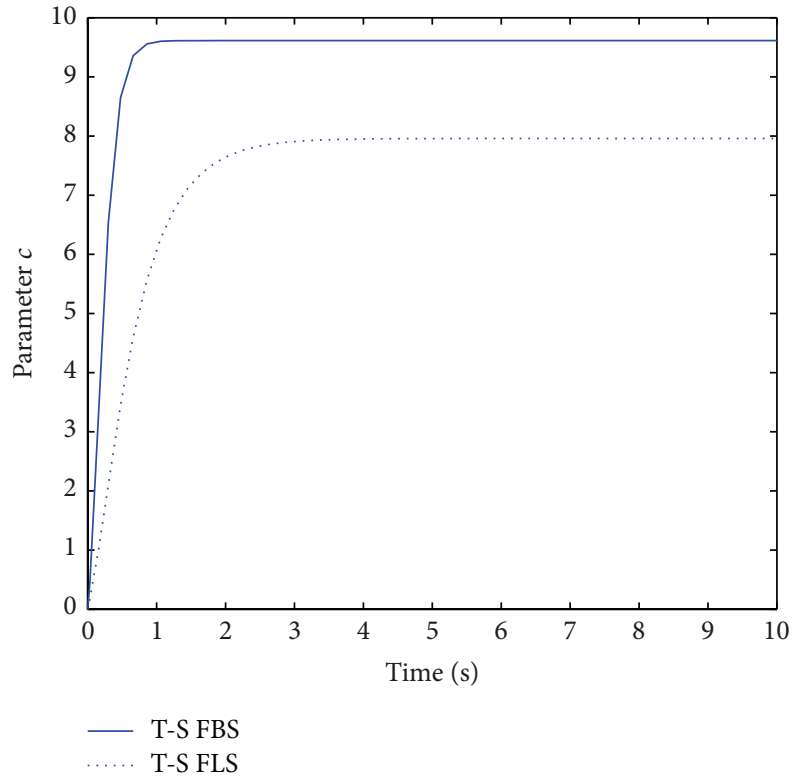

FIGURE 6: The curves of adaptive updated parameters.

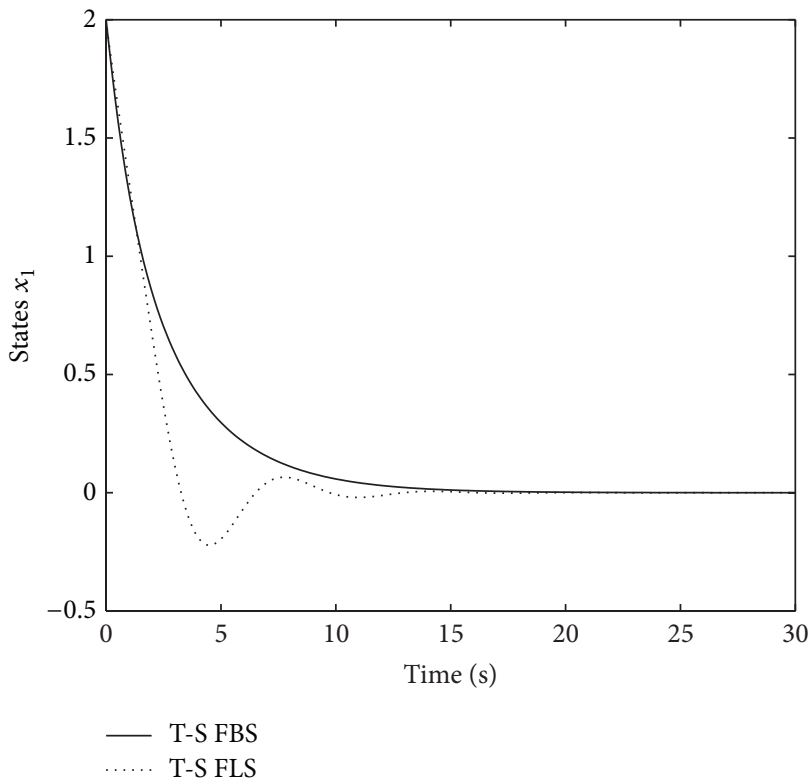

FIGURE 7: Responses of system state $x_{1}$ (T-S FBS: solid line, T-S FLS: dotted line).

are $x(0)=(2,-0.8)^{T}, \widehat{c}(0)=0$. The simulation results are shown in Figures 7, 8, 9, 10, and 11.

Through the comparison between T-S fuzzy linear model and bilinear one, we can see that the settling time of the systems is almost the same under the same initial conditions, although responses of T-S fuzzy bilinear system (FBS) state amplitudes are smaller than T-S fuzzy linear system (FLS), and the demand of the control input of the system is low. Thus, the proposed method has better dynamic performances than the existing ones based on T-S fuzzy linear model. 


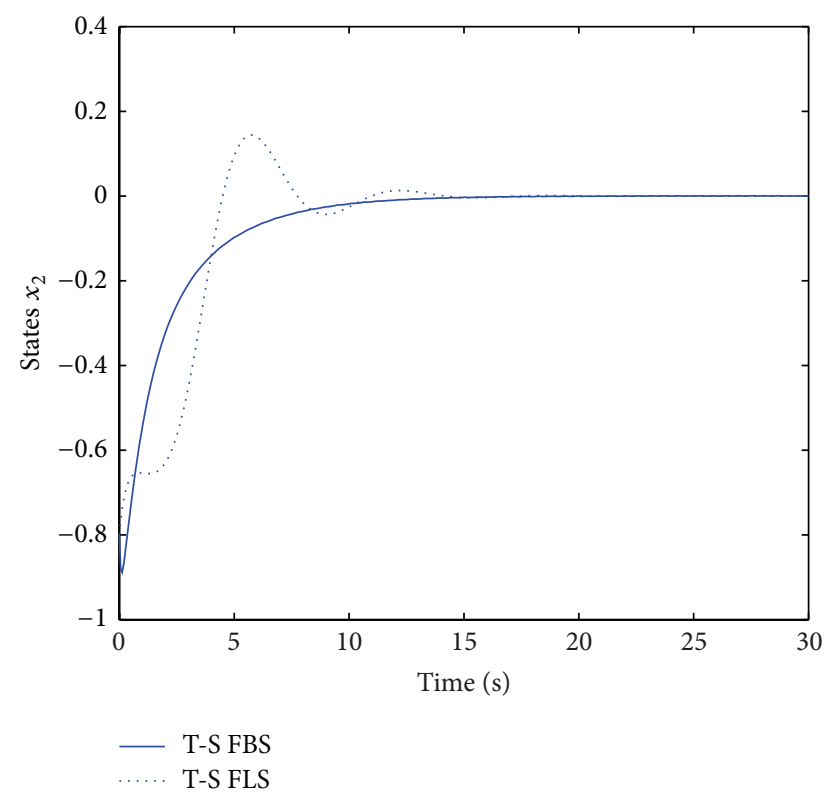

FIGURE 8: Responses of system state $x_{2}$ (T-S FBS: solid line, T-S FLS: dotted line).

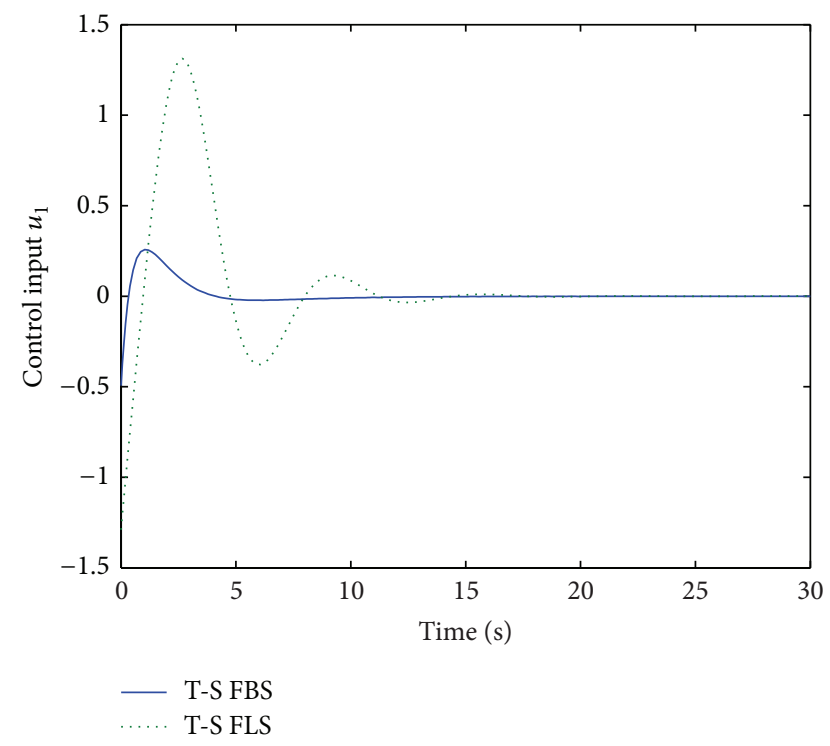

FIgURE 9: Control input $u_{1}$ (T-S FBS: solid line, T-S FLS: dotted line).

\section{Conclusion}

This paper proposes a new modelling method based on the multiple inputs T-S fuzzy bilinear model which is used to approximate nonlinear system; the parallel distributed compensation (PDC) method is utilized to design the fuzzy controller without considering the error caused by fuzzy modelling. The sufficient conditions with respect to decay rate $\alpha$ are derived by linear matrix inequalities (LMIs). The error caused by fuzzy modelling is considered and the method of adaptive control is used to reduce the effect of the modelling

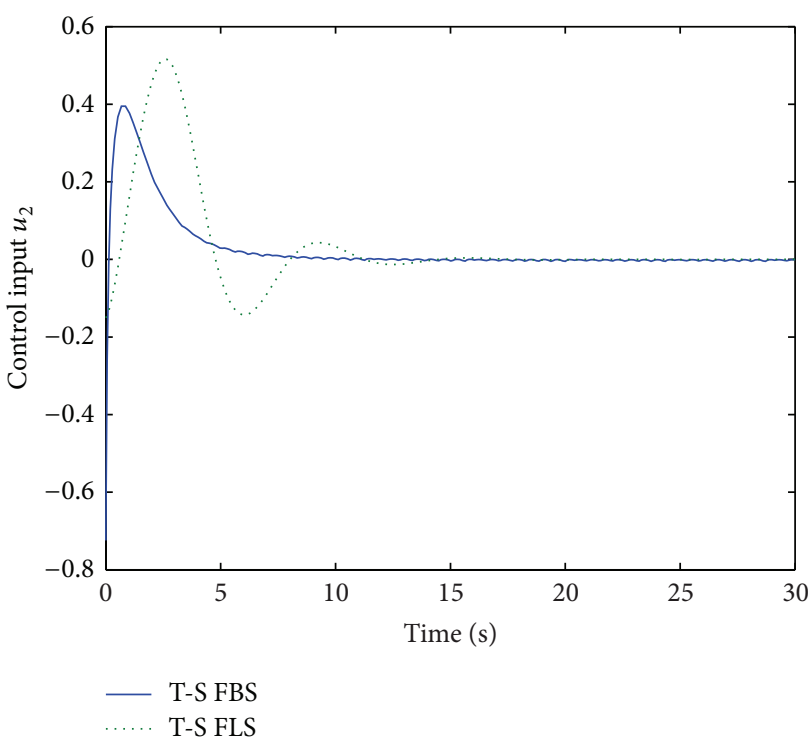

FIGURE 10: Control input $u_{2}$ (T-S FBS: solid line, T-S FLS: dotted line).

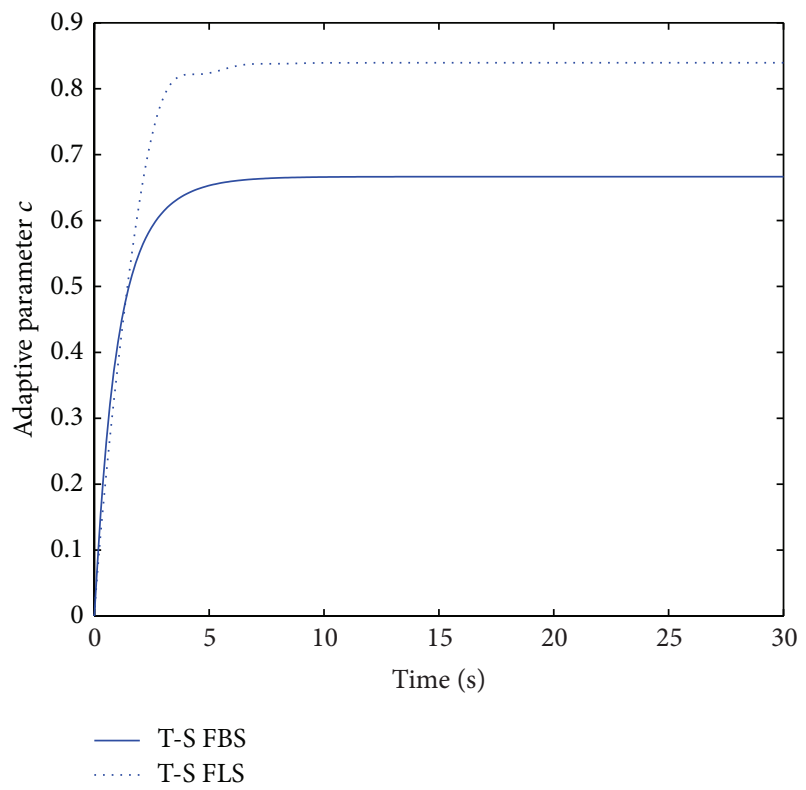

FIGURE 11: Adaptive parameter $c$ (T-S FBS: solid line, T-S FLS: dotted line).

error. By Lyapunov stability criterion, the resulting closedloop system is proved to be asymptotically stable. Finally, two illustrative examples are provided to show that the approach based T-S fuzzy bilinear systems have some advantages of performance over the existing methods based on T-S fuzzy linear system. The future research work is to extend the approach to general system, such as discrete-time systems, stochastic systems, and time-delay systems. 


\section{Conflict of Interests}

The authors declare that there is no conflict of interests regarding the publication of this paper.

\section{Acknowledgment}

This project was supported by the Soft Science Foundation of Shanxi Province (2011041033-3).

\section{References}

[1] K. Tanaka and H. O. Wang, Fuzzy Control Systems Design and Analysis: A Linear Matrix Inequality Approach, Wiley-Interscience, 2001.

[2] G. Feng, "A survey on analysis and design of model-based fuzzy control systems," IEEE Transactions on Fuzzy Systems, vol. 14, no. 5, pp. 676-697, 2006.

[3] K. Tanaka, T. Ikeda, and H. O. Wang, "Fuzzy regulators and fuzzy observers: relaxed stability conditions and LMI-based designs," IEEE Transactions on Fuzzy Systems, vol. 6, no. 2, pp. 250-265, 1998.

[4] E. Kim and H. Lee, "New approaches to relaxed quadratic stability condition of fuzzy control systems," IEEE Transactions on Fuzzy Systems, vol. 8, no. 5, pp. 523-534, 2000.

[5] K. Tanaka and M. Sugeno, "Stability analysis and design of fuzzy control systems," Fuzzy Sets and Systems, vol. 45, no. 2, pp. 135$156,1992$.

[6] H.-N. Wu and H.-X. Li, "New approach to delay-dependent stability analysis and stabilization for continuous-time fuzzy systems with time-varying delay," IEEE Transactions on Fuzzy Systems, vol. 15, no. 3, pp. 482-493, 2007.

[7] F. Zheng, Q.-G. Wang, and T. H. Lee, "Adaptive and robust controller design for uncertain nonlinear systems via fuzzy modeling approach," IEEE Transactions on Systems, Man, and Cybernetics, Part B: Cybernetics, vol. 34, no. 1, pp. 166-178, 2004.

[8] C. L. Hwang, "A novel Takagi-Sugeno-based robust adaptive fuzzy sliding-mode controller," IEEE Transactions on Fuzzy Systems, vol. 12, no. 5, pp. 676-687, 2004.

[9] S. Dong, Adaptive Fuzzy Control of Nonlinear System, Science and Technology Publishing House, Beijing, China, 2006.

[10] W.-Y. Wang, Y.-H. Chien, Y.-G. Leu, and T.-T. Lee, "Adaptive T-S fuzzy-neural modeling and control for general MIMO unknown non-affine nonlinear systems using projection update laws," Automatica, vol. 46, no. 5, pp. 852-863, 2010.

[11] Y.-H. Chien, W.-Y. Wang, Y.-G. Leu, and T.-T. Lee, "Robust adaptive controller design for a class of uncertain nonlinear systems using online T-S fuzzy-neural modeling approach," IEEE Transactions on Systems, Man, and Cybernetics, Part B: Cybernetics, vol. 41, no. 2, pp. 542-552, 2011.

[12] S. P. Moustakidis, G. A. Rovithakis, and J. B. Theocharis, "An adaptive neuro-fuzzy tracking control for multi-input nonlinear dynamic systems," Automatica, vol. 44, no. 5, pp. 1418-1425, 2008.

[13] K.-Y. Lian and H.-W. Tu, "LMI-Based adaptive tracking control for parametric strict-feedback systems," IEEE Transactions on Fuzzy Systems, vol. 16, no. 5, pp. 1245-1258, 2008.

[14] Z. Lendek, J. Lauber, T. M. Guerra, R. Babuka, and B. De Schutter, "Adaptive observers for TS fuzzy systems with unknown polynomial inputs," Fuzzy Sets and Systems, vol. 161, no. 15, pp. 2043-2065, 2010.
[15] C.-H. Hyun, C.-W. Park, and S. Kim, "Takagi-Sugeno fuzzy model based indirect adaptive fuzzy observer and controller design," Information Sciences, vol. 180, no. 11, pp. 2314-2327, 2010.

[16] Y.-H. Chang, W.-S. Chan, and C.-W. Chang, "T-S fuzzy modelbased adaptive dynamic surface control for ball and beam system," IEEE Transactions on Industrial Electronics, vol. 60, no. 6, pp. 2251-2263, 2013.

[17] S. T. Wang and J. T. Fei, "Robust adaptive sliding mode control of MEMS gyroscope using T-S fuzzy model," Nonlinear Dynamics, vol. 77, no. 1-2, pp. 361-371, 2014.

[18] R. Qi, G. Tao, C. Tan, and X. Yao, "Adaptive control of discretetime state-space T-S fuzzy systems with general relative degree," Fuzzy Sets and Systems, vol. 217, pp. 22-40, 2013.

[19] H. B. Jiang, J. J. Yu, and C. G. Zhou, "Stable adaptive fuzzy control of nonlinear systems using small-gain theorem and LMI approach," Journal of Control Theory and Applications, vol. 8, no. 4, pp. 527-532, 2010.

[20] R. R. Mohler, Bilinear Control Processes, Academic Press, New York, NY, USA, 1973.

[21] D. L. Elliott, Bilinear Systems in Encyclopedia of Electrical Engineering, Wiley, New York, NY, USA, 2001.

[22] T.-H. S. Li and S.-H. Tsai, "T-S fuzzy bilinear model and fuzzy controller design for a class of nonlinear systems," IEEE Transactions on Fuzzy Systems, vol. 15, no. 3, pp. 494-506, 2007.

[23] S. H. Tsai and T. H. S. Li, "Robust fuzzy control of a class of fuzzy bilinear systems with time-delay," Chaos, Solitons and Fractals, vol. 39, no. 5, pp. 2028-2040, 2009.

[24] T.-H. S. Li, S.-H. Tsai, J.-Z. Lee, M.-Y. Hsiao, and C.-H. Chao, "Robust $H_{\infty}$ fuzzy control for a class of uncertain discrete fuzzy bilinear systems," IEEE Transactions on Systems, Man, and Cybernetics, Part B: Cybernetics, vol. 38, no. 2, pp. 510-527, 2008.

[25] G. Zhang, J.-M. Li, and Y.-W. Ge, "Nonfragile guaranteed cost control of discrete-time fuzzy bilinear system with time-delay," Journal of Dynamic Systems, Measurement and Control, Transactions of the ASME, vol. 136, no. 4, Article ID 044502, 2014.

[26] J. R. Li, J. M. Li, and Z. L. Xia, "Observer-based fuzzy control design for discrete-time T-S fuzzy bilinear systems," International Journal of Uncertainty, Fuzziness and Knowledge-Based Systems, vol. 21, no. 3, pp. 435-454, 2013.

[27] M. S. Ali, "Robust stability of stochastic fuzzy impulsive recurrent neural networks with time varying delays," Iranian Journal of Fuzzy Systems, vol. 11, no. 4, pp. 1-13, 2014.

[28] M. Syed Ali, "Robust stability analysis of Takagi-Sugeno uncertain stochastic fuzzy recurrent neural networks with mixed time-varying delays," Chinese Physics B, vol. 20, no. 8, Article ID 080201, 2011. 


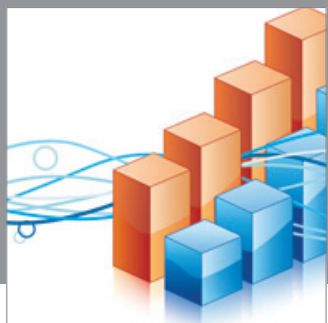

Advances in

Operations Research

mansans

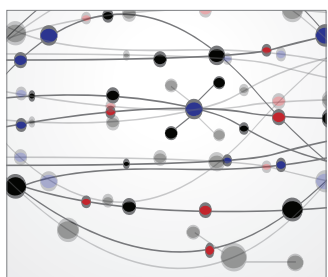

The Scientific World Journal
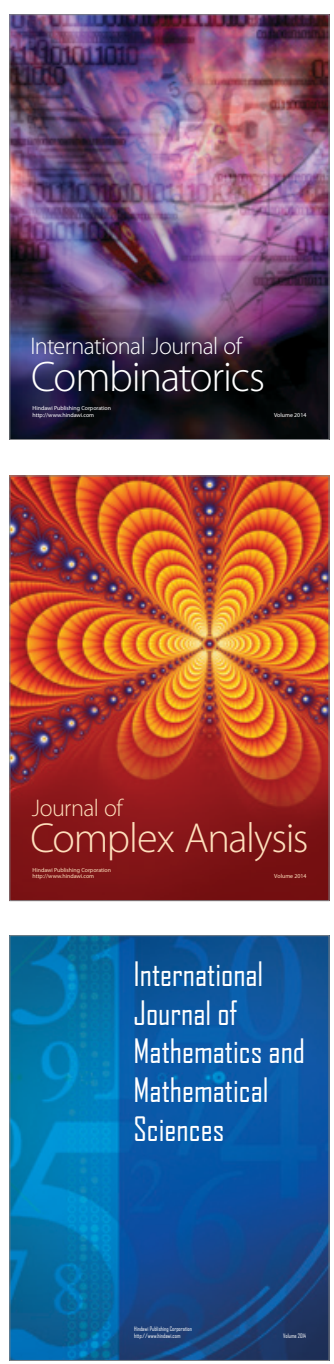
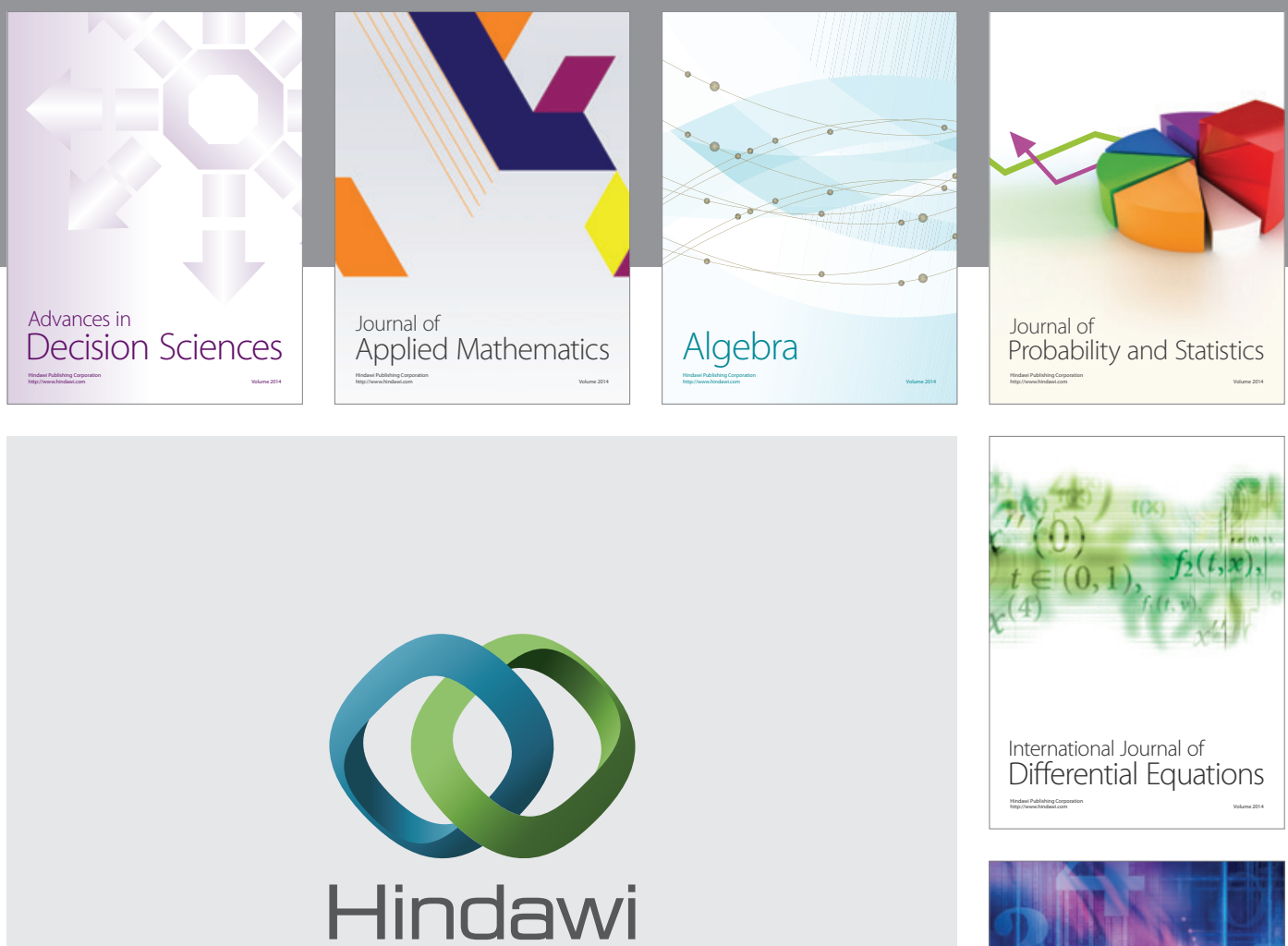

Submit your manuscripts at http://www.hindawi.com
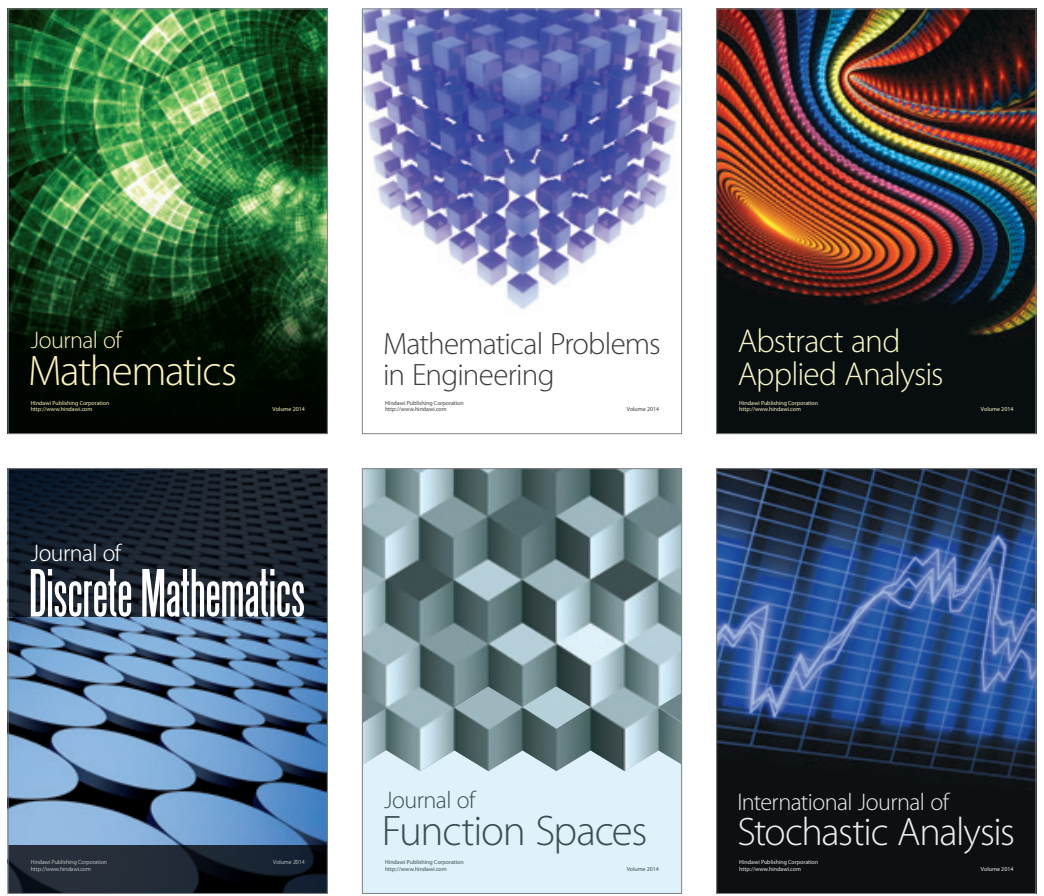

Journal of

Function Spaces

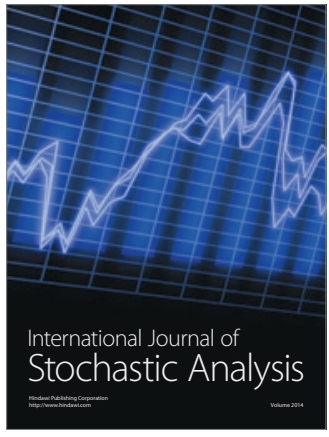

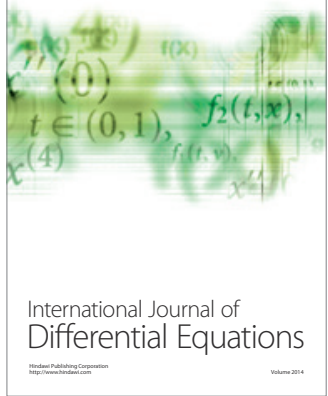
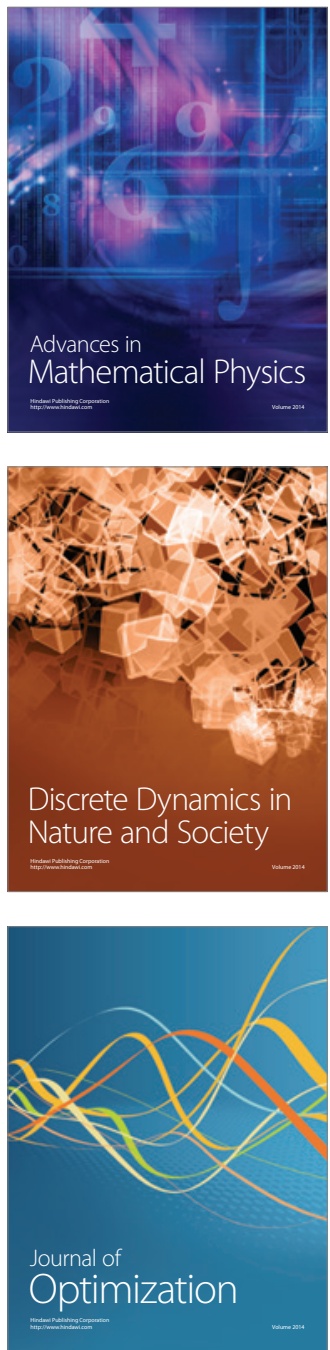